
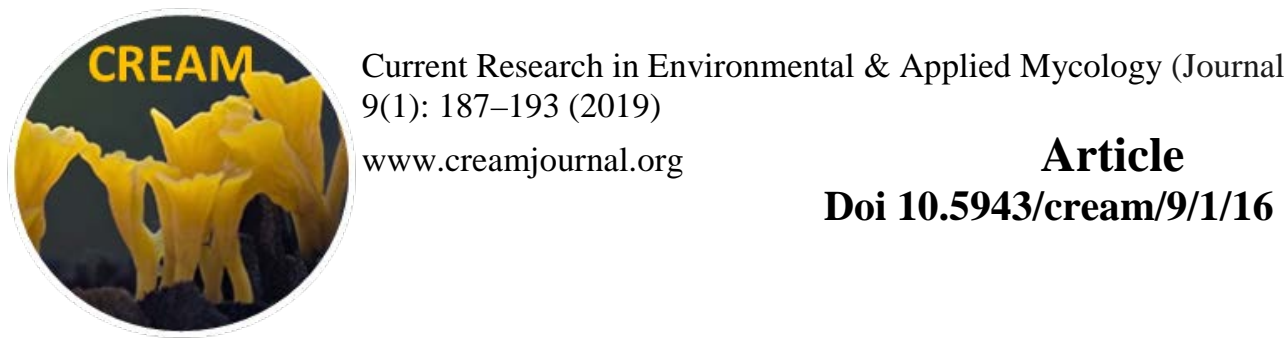

\title{
A second-order kinetic model on the survival profile of Candida albicans in biofilms
}

\section{Sumalapao DEP ${ }^{1,3,4, *}$, Alegre FMD $^{4,5}$, Salazar PBD $^{4,5}$, Villarante $\mathbf{N R}^{2}$, Gonzaga $\mathrm{AC}^{2}$ and Gloriani $\mathrm{NG}^{3}$}

\author{
${ }^{1}$ Department of Epidemiology and Biostatistics, College of Public Health, University of the Philippines Manila, Manila, \\ Philippines \\ ${ }^{2}$ Department of Physical Sciences and Mathematics, College of Arts and Sciences, University of the Philippines Manila, \\ Manila, Philippines \\ ${ }^{3}$ Department of Medical Microbiology, College of Public Health, University of the Philippines Manila, Manila, \\ Philippines \\ ${ }^{4}$ Biology Department, College of Science, De La Salle University, Manila, Philippines \\ ${ }^{5}$ College of Medicine, De La Salle Medical and Health Sciences Institute, Cavite, Philippines
}

Sumalapao DEP, Alegre FMD, Salazar PBD, Villarante NR, Gonzaga AC, Gloriani NG 2019 A second-order kinetic model on the survival profile of Candida albicans in biofilms. Current Research in Environmental \& Applied Mycology (Journal of Fungal Biology) 9(1), 187-193, Doi 10.5943/cream/9/1/16

\begin{abstract}
The prevalent use of indwelling medical devices has almost paralleled the increasing frequency of fungal infections commonly found in clinical practice. The present study examined Candida albicans biofilms on the surfaces of polyvinyl chloride (PVC) endotracheal tube, silicone urinary catheter, and silicone nasogastric tube. The viable C. albicans in biofilms was quantified using standard plating procedure. Several kinetic rate equations were employed to describe the survival profile of the viable population. Candida albicans exhibited remarkably heterogeneous growth patterns on the different medical devices reflecting variations on its adhesion potential and biofilm formation. A significant difference in the viability of $C$. albicans in biofilms on the surfaces of the medical devices was observed among monitoring points. Survival profile of $C$. albicans in biofilms followed a second-order kinetic model. Quantitative descriptions regarding growth patterns and kinetic profile of the fungus were obtained on these model biofilms. These findings can provide additional information to better understand the complex biology of $C$. albicans and to possibly explain the resistance patterns of fungal biofilms with the existing available antifungal drugs.
\end{abstract}

Key words - Fungal infections - Medical Devices - Polyvinyl chloride polymer - Silicone polymer - Survival analysis

\section{Introduction}

The prevalence of infections due to pathogenic microorganisms including Candida albicans has been increasing over the years (Pfaller \& Diekema 2007). Candida albicans has been observed to thrive in the skin, oral cavity and gastrointestinal tract, respiratory and genitourinary tracts particularly the vulvovaginal and perianal areas (Pfaller \& Diekema 2007). However, this fungus has been implicated in various infections particularly candidiasis which can progress from superficial to a more deleterious systemic infection (Rekha \& Visyasagar 2013). Increasing cases of candidiasis 
have been observed (Ghannoum \& Rice 1999) which was attributed to the use of various indwelling and implant devices (Richards et al. 1999, Shin et al. 2002, Taff et al. 2012). The surfaces of these clinically relevant devices provide substrates for cell adhesion and subsequent biofilm formation (Shin et al. 2002, Mohamed \& Al-Ahmadey 2013) resulting in nosocomial infections (Douglas 2003, El-Azizi et al. 2015). Currently, there has been no research conducted which assessed the viability profile of C. albicans in biofilms on the surfaces of medical devices. This study quantitatively characterized the kinetic relationship between the viable sessile fungal cells and time. These assessments provide a comparative analysis on the population dynamics of C. albicans on medical devices with different chemical compositions. Hence, the present study quantified the viable $C$. albicans population in the biofilms on PVC endotracheal tube, silicone urinary catheter, and silicone nasogastric tube surfaces using standard plating procedure. Furthermore, several kinetic rate equations were employed to describe the growth profile of the viable population. Quantitative descriptions including growth patterns and kinetic profile of $C$. albicans were obtained on these model biofilms on the surfaces of different clinically important medical devices. Findings of this study can provide additional information for a better understanding of the complex biology of $C$. albicans and some possible explanations on the resistance patterns of fungal biofilms with the existing available antimycotics.

\section{Materials \& Methods}

\section{Biofilm formation of Candida albicans on medical devices}

An isolate of Candida albicans (ATCC 14053) was given by the University of the Philippines Manila (Department of Medical Microbiology, College of Public Health). Subsequently, the organism was grown on Sabouraud dextrose agar (SDA) plates $\left(48 \mathrm{~h}\right.$ incubation at $37^{\circ} \mathrm{C}$ ) prior to infection. The inoculum was prepared following the protocol of Andes et al. (2004) with slight modifications. Three distinct colonies were suspended into $10 \mathrm{~mL}$ sterile distilled water. A serial dilution technique was applied to determine the density of the inoculum and its viable fungal count was confirmed using SDA.

Modified methods of Hawser \& Douglas (1994) and Chandra et al. (2001) were employed for growing biofilms. Disks $\left(0.16 \mathrm{~cm}^{2}: 0.2 \mathrm{~cm}\right.$ x $\left.0.8 \mathrm{~cm}\right)$ from the PVC endotracheal tube (ID: $7.5 \mathrm{~mm}$, OD: $11.1 \mathrm{~mm}$, Sacett, Portex), silicone urinary catheter (Fr18, Surgitech+, Fujian Bestway Medical Polymer Corp.), and silicone nasogastric tube (Fr5, Medline ${ }^{\circledR}$, NeoMed, Inc.) were prepared. The prepared disks were sterilized, oven-dried, and individually placed in 96-well microtiter plates. The disks were charged with inoculum $(100 \mu \mathrm{L})$ and $50 \mathrm{mM}$ glucose $(200 \mu \mathrm{L})$ and then incubated at $37^{\circ} \mathrm{C}$ for a period of $72 \mathrm{~h}$ to allow biofilm formation.

\section{Viability and kinetic profile of Candida albicans in biofilms}

Since fungal biofilm formation has been observed as early as $4 \mathrm{~h}$ (Sumalapao et al. 2018), serial monitoring (4-72 h) of the viable population of C. albicans in biofilm was done. Microscopic examination of the biofilm formation was performed. For the viability evaluation (Sumalapao et al. 2018), the disks were washed with $0.15 \mathrm{M}$ PBS ( $5 \mathrm{~mL}$ ). To remove adherent sessile cells, disks were immersed into a $10 \mathrm{~mL}$ sterile distilled water, vortexed for $15 \mathrm{~s}$. To quantify the viable sessile fungal count, the suspension was plated on SDA and incubated at $37^{\circ} \mathrm{C}$ for $24 \mathrm{~h}$. Each test was performed in triplicates. The results were reported as mean \pm standard deviation $\left(\log _{10} \mathrm{CFU} / \mathrm{mL}\right)$.

\section{Numerical calculations and statistical analysis}

Viable fungal counts in biofilms were compared using analysis of variance and Bonferroni test. To elucidate the growth rate profile of C. albicans, several kinetic equations (Sumalapao et al. 2017) including zero-order, first-order, and second-order models were examined (Table 1). All statistical assessments were calculated using STATA ${ }^{\odot}$ V12.0 at 5\% significance level. 
Table 1 Survival kinetic model equations.

\begin{tabular}{lll}
\hline Order & Rate Equation & Linearized Form \\
\hline Zero & $\frac{d P_{t}}{d t}=c_{o}$ & $P_{t}=c_{0} t+P_{o}$ \\
First & $\frac{d P_{t}}{d t}=c_{1} P_{t}$ & $\log P_{t}=\log P_{o}+\frac{c_{1}}{2.303} t$ \\
Second & $\frac{d P_{t}}{d t}=c_{2} P_{t}{ }^{2}$ & $\frac{-1}{P_{t}}=c_{2} t+\left(\frac{-1}{P_{0}}\right)$
\end{tabular}

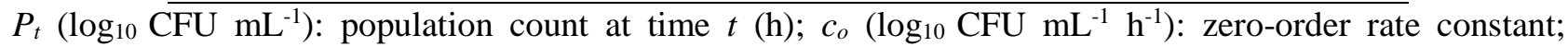

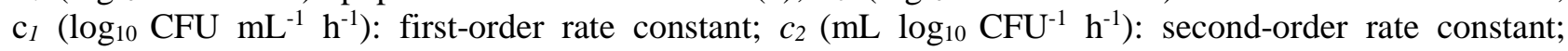
CFU: colony forming unit

\section{Results}

Microscopic examination of $C$. albicans biofilms on the surfaces of medical devices was performed (Fig. 1). The fungus exhibited remarkable heterogeneous growth patterns on the different medical devices reflecting variations on its adhesion potential and biofilm formation. The viable $C$. albicans in biofilms was quantified using standard plating procedure (Fig. 2). Results on the viable count monitoring of $C$. albicans are presented in Table 2. This study has identified that the fungal population increased as the biofilms formed over time. In PVC endotracheal tube, the mean viable population counts on the 24, 36, 48, 60, and 72 h significantly varied from the 4 and $8 \mathrm{~h}$ population counts $(p<0.05)$. Moreover, viability counts on the 24 -h biofilm significantly differed when compared with 48,60 , and $72 \mathrm{~h}$ biofilms $(p<0.05)$, and the viable population counts on $48 \mathrm{~h}$ also significantly differed from the 60 and $72 \mathrm{~h}$ biofilm formation $(p<0.05)$. For the silicone urinary catheter, a significant change in the viable fungal counts was observed from 36-h biofilm formation when compared with the 4 and 8 h population counts $(p<0.05)$. The viable population counts from $36 \mathrm{~h}$ did not significantly differ when compared with the viable counts in the subsequent monitoring points. Viable C. albicans population counts on the surface of silicone nasogastric tube significantly differed every 12-h interval from 12 to $60 \mathrm{~h}$ biofilm formation, but the population counts did not differ on the 60 and $72 \mathrm{~h}$ biofilm formation (Table 2).
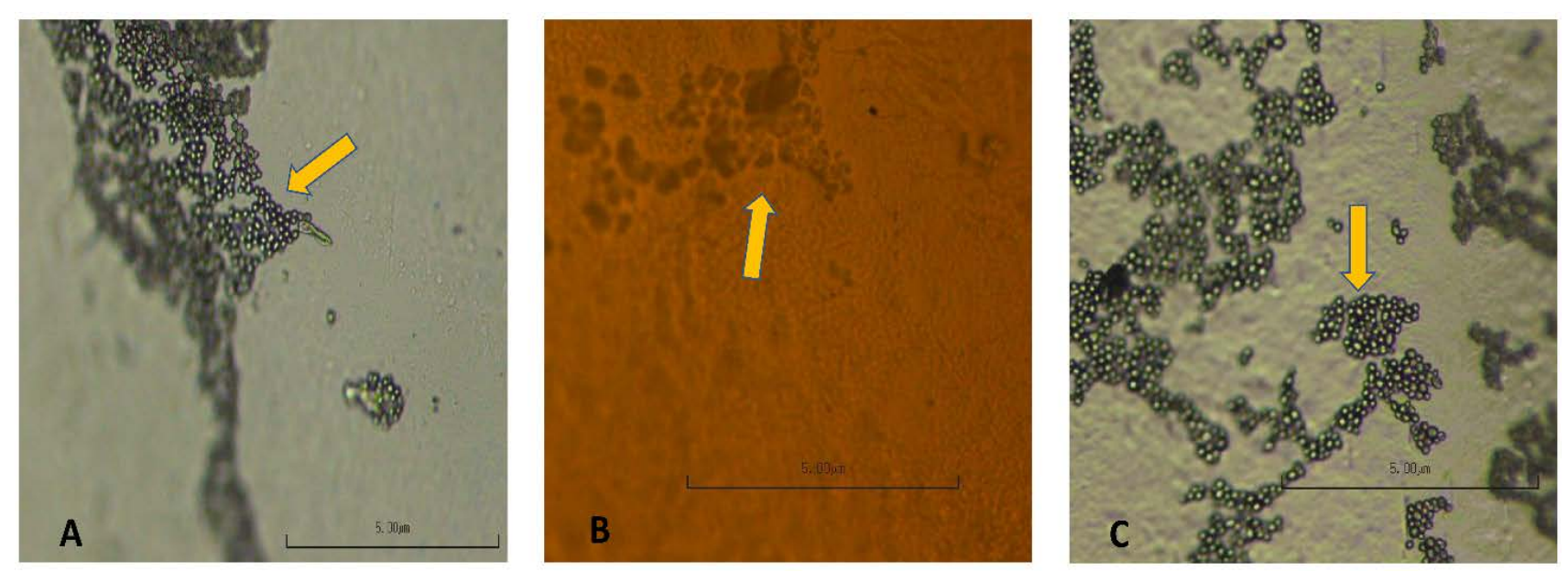

Fig. 1 - Micrographs of Candida albicans biofilms on (A) polyvinyl chloride endotracheal tube, (B) silicone urinary catheter, and (C) silicone nasogastric tube surfaces. Structures in yellow arrows indicate 24-h biofilm formation viewed using light microscopy at high power objective. 


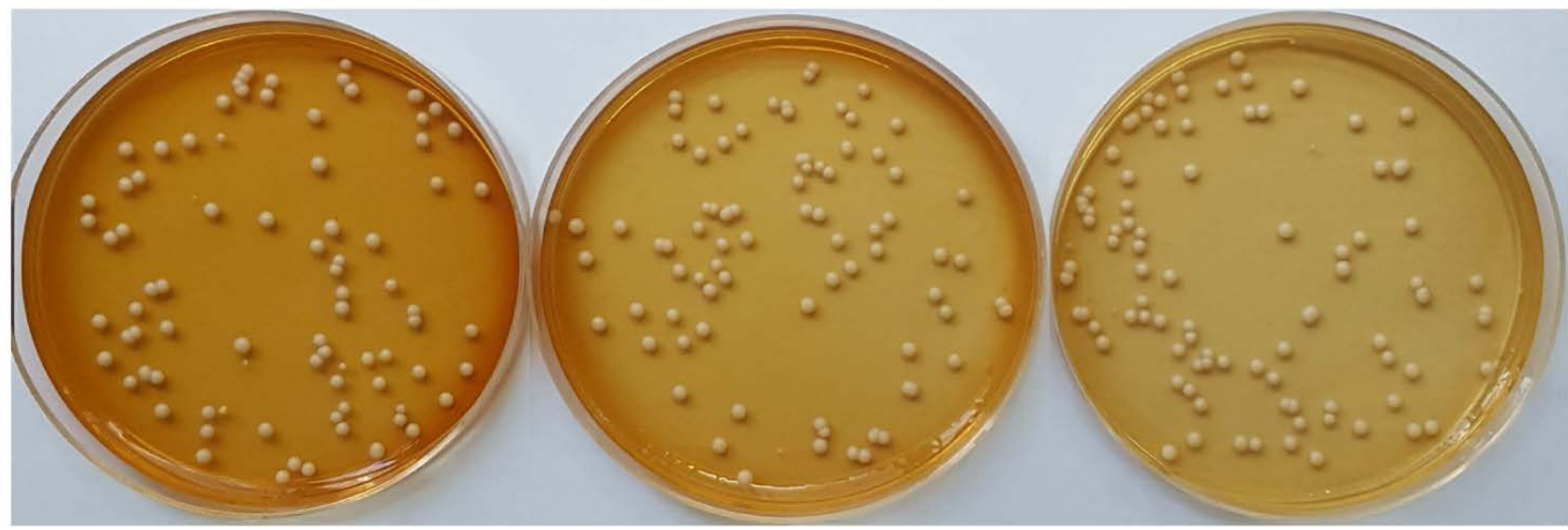

Fig. 2 - Morphological appearance of Candida albicans colonies in Sabouraud dextrose agar plates, $24 \mathrm{~h}$ incubation at $37^{\circ} \mathrm{C}$.

Table 2 Viable Candida albicans cell count in biofilms on the surfaces of different medical devices at different monitoring intervals.

Viable population count (mean \pm standard deviation, $\log _{10} \mathrm{CFU} / \mathrm{mL}$ )

\begin{tabular}{llll} 
Time (h) & $\begin{array}{l}\text { Polyvinyl chloride } \\
\text { endotracheal tube }\end{array}$ & $\begin{array}{l}\text { Silicone urinary } \\
\text { catheter }\end{array}$ & $\begin{array}{l}\text { Silicone nasogast } \\
\text { tube }\end{array}$ \\
\hline 4 & $2.985 \pm 0.009^{\mathrm{a}}$ & $3.780 \pm 0.032^{\mathrm{a}}$ & $3.139 \pm 0.008^{\mathrm{a}}$ \\
8 & $3.028 \pm 0.024^{\mathrm{a}}$ & $3.787 \pm 0.029^{\mathrm{a}}$ & $3.156 \pm 0.009^{\mathrm{ab}}$ \\
12 & $3.048 \pm 0.011^{\mathrm{ab}}$ & $3.806 \pm 0.027^{\mathrm{ab}}$ & $3.168 \pm 0.007^{\mathrm{b}}$ \\
24 & $3.132 \pm 0.049^{\mathrm{bc}}$ & $3.832 \pm 0.026^{\mathrm{ab}}$ & $3.351 \pm 0.010^{\mathrm{c}}$ \\
36 & $3.226 \pm 0.020^{\mathrm{cd}}$ & $3.893 \pm 0.036^{\mathrm{bc}}$ & $3.395 \pm 0.005^{\mathrm{d}}$ \\
48 & $3.304 \pm 0.016^{\mathrm{d}}$ & $3.964 \pm 0.045^{\mathrm{c}}$ & $3.430 \pm 0.003^{\mathrm{e}}$ \\
60 & $3.799 \pm 0.054^{\mathrm{e}}$ & $3.968 \pm 0.034^{\mathrm{c}}$ & $3.849 \pm 0.007^{\mathrm{f}}$ \\
72 & $3.812 \pm 0.040^{\mathrm{e}}$ & $3.967 \pm 0.038^{\mathrm{c}}$ & $3.849 \pm 0.013^{\mathrm{f}}$ \\
\hline
\end{tabular}

Values containing similar superscript letters in a given column do not differ at $5 \%$ significance level. CFU: colony forming units

When the kinetic survival of C. albicans biofilm on the surfaces was assessed using the zeroorder rate equation, the viable fungal counts on the surface of silicone urinary catheter behaved in accordance to this model $\left(R^{2}=0.9347\right.$, Table 3$)$. The doubling time of the fungal population in biofilm is approximately $102 \mathrm{~h}(4.25 \mathrm{~d})$ under the zero-order kinetic model. Further examination on the survival profile of fungal populations, the first-order kinetic model generated a better fit of the experimental data as justified by higher coefficients of determination and smaller error values (Table 3). Fungal biofilm populations are expected to have doubling time of $1 \mathrm{~d}$ for PVC endotracheal tube, $4 \mathrm{~d}$ for silicone urinary catheter, and $11 \mathrm{~d}$ for silicone nasogastric tube under the first-order kinetic rate equation. However, when the viability of C. albicans in biofilms was examined using the secondorder kinetic model, estimates of the parameters showed coefficients of determination $\left(R^{2}\right)$ closest to unity: 0.9266, 0.9354 , and 0.9359 for PVC endotracheal tube, silicone urinary catheter, and silicone nasogastric tube, respectively (Table 3). These findings suggest that the survival of C. albicans in biofilms on medical devices behaved in accordance with the second-order kinetic model. These results are further justified by the smallest sum of squares of the error measures and lowest $p$-values (Table 3). 
Table 3 Estimates of the parameters describing the survival of Candida albicans in biofilms on the surface of medical devices.

\begin{tabular}{lllll}
\hline Kinetic Model & Parameter & $\begin{array}{l}\text { Polyvinyl chloride } \\
\text { endotracheal tube }\end{array}$ & $\begin{array}{l}\text { Silicone urinary } \\
\text { catheter }\end{array}$ & $\begin{array}{l}\text { Silicone } \\
\text { nasogastric tube }\end{array}$ \\
\hline \multirow{5}{*}{ Zero-order } & $c_{0}$ & 82.6051 & 56.3283 & 86.6085 \\
& $P_{o}$ & -95.5516 & $5.77 \mathrm{e} 3$ & $3.71 \mathrm{e} 2$ \\
& $R^{2}$ & 0.7809 & 0.9347 & 0.8179 \\
& $S S E$ & $8.56 \mathrm{e} 6$ & $9.91 \mathrm{e} 5$ & $7.47 \mathrm{e} 6$ \\
& $P$ & 0.0036 & 0.0001 & 0.0020 \\
\hline \multirow{5}{*}{ First-order } & $c_{1}$ & 0.0289 & 0.0074 & 0.0025 \\
& $P_{O}$ & $7.56 \mathrm{e} 2$ & $5.88 \mathrm{e} 3$ & $1.34 \mathrm{e} 3$ \\
& $R^{2}$ & 0.8981 & 0.9357 & 0.9185 \\
& $S S E$ & 0.0799 & 0.0032 & 0.0476 \\
Second-order & $P$ & 0.0003 & 0.0001 & 0.0002 \\
& $c_{2}$ & -0.0011 & -0.0002 & -0.0009 \\
& $P_{O}$ & 2.9181 & 3.7711 & 3.0823 \\
& $R^{2}$ & 0.9266 & 0.9354 & 0.9359 \\
& $S S E$ & $4.31 \mathrm{e}-4$ & $1.40 \mathrm{e}-5$ & $2.54 \mathrm{e}-4$ \\
\hline
\end{tabular}

$c_{o}$ : zero-order constant; $c_{1}$ : first-order constant; $c_{2}$ : second-order constant; SSE: sum of squares of the error; $p$ : p-value; $R^{2}$ : coefficient of determination; $P_{0}$ : initial population.

\section{Discussion and Conclusion}

Candida is the most common genus of fungi that colonizes medical devices by forming resilient biofilm (Desai et al. 2014). The increasing use of implanted medical devices enhances the deviceassociated infections, including fungal biofilm formation. With more than one million reported cases of nosocomial infections resulting from the prevalent use of urinary catheters (Nett et al. 2014), Candida spp. were identified as the causative agents of at least $25 \%$ of these urinary infections (Fisher et al. 2011). In intensive care unit patients, the most common infective nosocomial cause of mortality is ventilator-associated pneumonia (Mariyaselvam et al. 2017), and the typical fungus found on the surface of the PVC endotracheal tubes used in ventilation is C. albicans (Machado \& Webster 2017). Candida albicans adheres on the surface of medical devices including silicone nasogastric tubes which are used for patients with complications such as poor voluntary intake or gut dysfunction to facilitate nutrient uptake (Blumenstein et al. 2014). In this study, we have presented that the growth of C. albicans in biofilms on the surfaces of different medical devices has a survival kinetic profile in accordance with the second-order rate equation. The organism exhibited faster adhesion and subsequent proliferation on the surface of silicone urinary catheter when compared to that of PVC endotracheal tube and silicone nasogastric tube. In general, the growth profile exhibited a linear pattern until $24 \mathrm{~h}$ after inoculation of $C$. albicans and increased exponentially from 24 to $48 \mathrm{~h}$. These quantitative assessments provide a comparative analysis on the population dynamics of $C$. albicans on medical devices with different chemical compositions. Candida albicans has been shown to survive in the biofilms on the surfaces of medical devices and their counts increased slightly over time. Our study has identified the increasing viable fungal population which behaved in a nonlinear manner simulating an exponential growth under a second-order kinetic equation.

In the assessment of the viability of the fungal population on these biofilms, our study employed the standard plate method. In most researches, plate counting is used frequently in assessing the viability of microorganisms (Sumalapao et al. 2017). However, with the cell viability complex nature, whether cells are culturable or not in the chosen growth medium and specified conditions (Sumalapao et al. 2017), fungal cells that are not culturable in vitro but are active, can still have some possible detrimental health effects. Hence, there is a need for a more advanced and appropriate method to explore. Due to the complex effects of diversed fungal cell populations, an 
advanced method can provide more accurate viability quantification, generate better parameter estimates, and have more defined descriptions of the fungal population dynamics.

In conclusion, C. albicans biofilms on the surfaces of different medical devices have a complex architecture and heterogeneous structure. A significant difference in the viability of C. albicans in biofilms on the surfaces of the medical devices was observed among monitoring points. Growth profile on the survival of $C$. albicans followed a second-order kinetic model. The fungus exhibited remarkable heterogeneous growth patterns when monitored in varying medical devices reflecting its variability on adhesion potential, biofilm morphology, and survival profile. Furthermore, this study on biofilms can provide additional information for a better understanding of C. albicans complex biology and some plausible explanations on its resistance patterns with the existing available antifungal drugs.

\section{Acknowledgement}

Dr. Sumalapao is grateful for the financial assistance through the DOST-ASTHRDP Dissertation Grant. The authors extend their gratitude to Prof. Marohren Altura and Ms. Mary Ann Sison of the Department of Medical Microbiology, College of Public Health, University of the Philippines Manila for the isolates of Candida albicans.

\section{References}

Andes D, Nett J, Oschel P, Albrecht R et al. 2004 - Development and characterization of an in vivo central venous catheter Candida albicans biofilm model. Infection and Immunity 72, 60236031.

Blumenstein I, Shastri YM, Stein J. 2014 - Gastroenteric tube feeding: techniques, problems and solutions. World Journal of Gastroenterology 20, 8505-8524.

Chandra J, Kuhn DM, Mukherjee PK, Hoyer LL et al. 2001 - Biofilm formation by the fungal pathogen Candida albicans: development, structure, and drug resistance. Journal of Bacteriology 183, 5385-5394.

Desai JV, Mitchell AP, Andes DR. 2014 - Fungal biofilms, drug resistance, and recurrent infection. Cold Spring Harbor Perspectives in Medicine 4, 1-18.

Douglas LJ. 2003 - Candida biofilms and their role in infection. Trends in Microbiology 11, 30-36.

El-Azizi M, Farag N, Khardori N. 2015 - Antifungal activity of amphotericin and voriconazole against the biofilms and biofilm-dispersed cells of Candida albicans employing a newly developed in vitro pharmacokinetic model. Annals of Clinical Microbiology and Antimicrobials 14, 1-9.

Fisher J, Kavanagh K, Sobel J, Kauffman C, Newman C. 2011 - Candida urinary tract infection: pathogenesis. Clinical Infectious Diseases 52, 437-451.

Ghannoum MA, Rice LB. 1999 - Antifungal agents mode of action, mechanism of resistance and correlation of these mechanisms with bacterial resistance. Clinical Microbiology Reviews 12, 501-517.

Hawser SP, Douglas LJ. 1994 - Biofilm formation by Candida species on the surface of catheter materials in vitro. Infection and Immunity 62, 915-921.

Machado M, Webster T. 2017 - Lipase degradation of plasticized polyvinyl chloride endotracheal tube surface to create nanoscale features. International Journal of Nanomedicine 1, 2109-2115.

Mariyaselvam MZ, Marsh LL, Bamford S, Smith A et al. 2017 - Endotracheal tubes and fluid aspiration: an in vitro evaluation of new cuff technologies. BMC Anesthesiology 17, 1-9.

Mohamed SA, Al-Ahmadey ZZ. 2013 - Biofilm formation and antifungal susceptibility of Candida isolates from various clinical specimens. British Microbiology Research Journal 3, 590-601.

Nett JE, Brooks EG, Cabezas-Olcoz J, Sanchez H et al. 2014 - Rat indwelling urinary catheter model of Candida albicans biofilm infection. Infection and Immunity 82, 4931-4940.

Pfaller MA, Diekema DJ. 2007 - Epidemiology of invasive candidiasis: a persistent public health problem. Clinical Microbiology Reviews 20, 133-163. 
Rekha S, Visyasagar GM. 2013 - Anti-candida activity of medicinal plants: a review. International Journal of Pharmacy and Pharmaceutical Sciences 5, 9-16.

Richards MJ, Edwards JR, Culver DH, Gaynes RP. 1999 - Nosocomial infections in medical intensive care units in the United States. National Nosocomial Infections Surveillance System. Critical Care Medicine 27, 887-892.

Shin JH, Kee SJ, Shin MG, Kim SH et al. 2002 - Biofilm production by isolates of Candida species recovered from nonneutropenic patients: comparison of bloodstream isolates with isolates from other sources. Journal of Clinical Microbiology 40, 1244-1248.

Sumalapao DEP, Mesina JART, Cabrera EC, Gloriani NG. 2017 - Viability kinetics of Lactobacillus casei Shirota strain in a commercial fermented milk drink during refrigerated storage. National Journal of Physiology, Pharmacy and Pharmacology 7, 1242-1246.

Sumalapao DEP, Cabrera EC, Flores MJC, Amalin DM et al. 2018 - Viability kinetic profile, morphological structure, and physicochemical characterization of Candida albicans biofilm on latex silicone surfaces. Annual Research and Review in Biology 24, 1-8.

Taff HT, Nett JE, Andes DR. 2012 - Comparative analysis of Candida biofilm quantitation assays. Medical Mycology 50, 214-218. 